\title{
Design of Specialized Vocabulary Management System for Accounting English
}

\author{
Lifeng Wang ${ }^{1,}$, Yiyun Wang ${ }^{2, b}$ \\ ${ }^{1}$ Teaching and Research Institute of Foreign Language, Bohai University, Jinzhou, P.R. China \\ ${ }^{2}$ College of Management, Jilin University, Changchun, P.R. China \\ abhdxwanglifeng@163.com, byyw_jlu@163.com
}

\begin{abstract}
Keywords: Accounting English; J2EE architecture; overall design; management system; specialized vocabulary
\end{abstract}

\begin{abstract}
Accounting English as an international business language, its importance is increasingly apparent, because in terms of vocabulary its characteristics is particularly unique, so in order to facilitate memory and use specialized vocabulary designed accounting English specialized vocabulary management system is important. Firstly studied the frame structure used by vocabulary management system, namely J2EE architecture and theoretical knowledge; secondly describes the overall design of the system, including system design ideas and functional modules; finally based on the overall design of the system to detail design, including design principles as well as the specific features of each module. Design of accounting English vocabulary management system is not only easy to use, but also greatly improves the learning efficiency of vocabulary, for learning the English vocabulary has an important role.
\end{abstract}

\section{Introduction}

With the development of economic globalization and China's accession to WTO, accounting also faces challenges, which is to accounting personnel served in the foreign sphere put forward higher requirements. With the accelerated process of globalization, accounting English as the common language of international trade exchanges plays an important intermediary role. More and more accountants are asked to learn and master English in the accounting profession, with English solved accounting work. Accounting English has its own features, apart from jargon and special syntax it also includes invoices expressed by English special form, such as accounting documents, account books and so on. Therefore, the study of accounting English, mastered the accounting English language features and integration of accounting knowledge and English, which can improve our ability of reading English literature and the ability of treating accounting business with English [1].

The ultimate goal of accounting English is that English as a carrier to achieve the exchange and dissemination of accounting theory method. Accounting English as a branch of ESP it is obviously different from other English. Accounting English has the following characteristics: Accounting English vocabulary has unity, unity primarily refers within a particular subject area, a term only represents a specific meaning; highly specialized vocabulary and learning threshold is high, mainly due to the accounting is a highly specialized one discipline; specialized vocabulary has ambiguity, the meaning of ambiguity refers to that the concept represented words is a contradiction or association in logic; the extensive use of the prefix; the using of partial specialized vocabulary abbreviations. Learning English accounting vocabulary features helps to effectively learn and use accounting English, thus accounting English vocabulary management system designed in this paper has a significant role [2].

\section{J2EE Architecture}

Accounting English vocabulary management system studied in this paper mainly uses C/S, B/S hybrid structure to achieve system and uses J2EE framework technology to design. J2EE is architecture which using Java 2 Platform to simplify complex issues related to the multi-level 
enterprise solution development, deployment and management. The basis of J2EE technology is the core Java platform or the standard version of Java 2 Platform, J2EE not only consolidated many advantages in the standard edition, such as feature that "write once, run anywhere", JDBC API and CORBA technology which conveniently access to the database and security model with the ability to protect data and so on in Internet applications, meanwhile it also provides full support for EJB (Enterprise JavaBeans), Java Servlets API, JSP (Java Server Pages) and XML technology [3].

J2EE architecture provides an intermediate layer integrated framework to meet the needs that require high availability, high reliability and scalability of applications. Typically these are achieved by the distributed application, including the front data and back data source as well as a layer or layers among them; the intermediate layer provides the function that combines with business functions, data and EIS (Enterprise Information System). The intermediate layer separated client from the complex business logic, using sophisticated INTERNET technology makes time spent in the management minimized for users. By providing a unified development platform, J2EE reduces the cost and complexity of developing multi-tier application, meanwhile provides strong support for existing application integration; enhances security and improves performance [4]. Its architecture diagram is shown in Fig. 1.

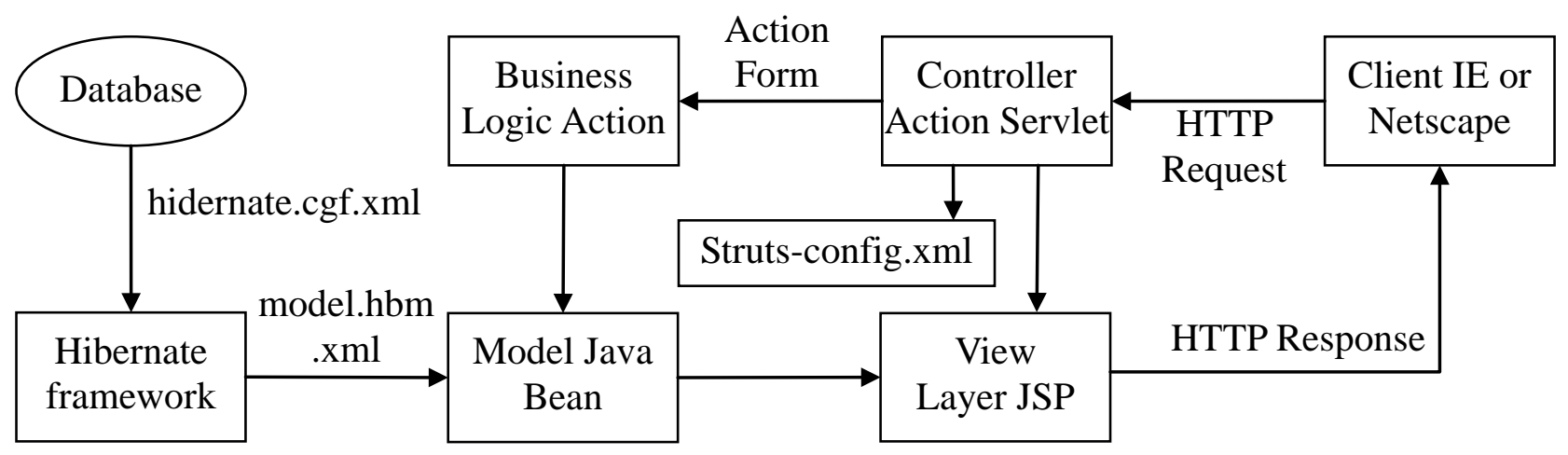

Fig. 1. J2EE architecture diagram

In which, view can be interpreted as User Interface Logic Layer (UI). View layer is responsible for displaying the results of the response and the carrier of response results has two forms: static page and dynamic page. In the Application Server the static page that is ordinary HTML pages. The most commonly used by the dynamic page is JSP (Java Server Page). Control layer is the dispatch centre of Application Server, mainly composed by the Servlet. Servlet is translated into server applet which is a cross-platform and protocol-independent service side component, it can achieve remote dynamic load on the network. Activation and movement of Servlet must have a client to call. Model layer accepts requests from clients and the results returns the client. The model layer is the hierarchy that concretely realizes the business processing functions, for Servlet calling, analysing and processing requests from clients, it can be subdivided into the business logic layer and the persistence layer [5].

\section{Overall Design}

The overall design is also called the outline design, namely the design for the global problem, which is the design of the system overall treatment program. In software engineering summary design includes computer configuration design, system module design, database and document design, code design and system reliability and internal control design etc. [6]. The overall design idea of accounting English vocabulary management system includes the following aspects: system consists of multiple modules, these modules are managed in a centralized form; using the idea of modular system design and making the system have the scalability and operability; rational database design, in the process of designing application system, among the various modules connected to each other by way of data stream, so the coupling degree is lower between the module and the module, and facilitate system proper operation, making the security of the system increase [7]. 
System design studied in this paper is mainly functional modular design of the system. Accounting English specialized vocabulary management system includes vocabulary query, the thesaurus manage, vocabulary translation, system settings and vocabulary study five modules. In which vocabulary query includes Chinese query, English query, history recording, favorites vocabulary four sub-modules; thesaurus management module includes adding vocabulary, deleting vocabulary, modify vocabulary, thesaurus updating four sub-module; vocabulary translation module includes Chinese to English, English to Chinese, detecting language, update meaning four sub-module; system settings module includes system maintenance, system introduction, font setting, time setting four sub-modules; vocabulary study module includes self testing, vocabulary memorization, word recording, vocabulary practice four sub-module. Its functional block diagram is shown in Fig. 2.

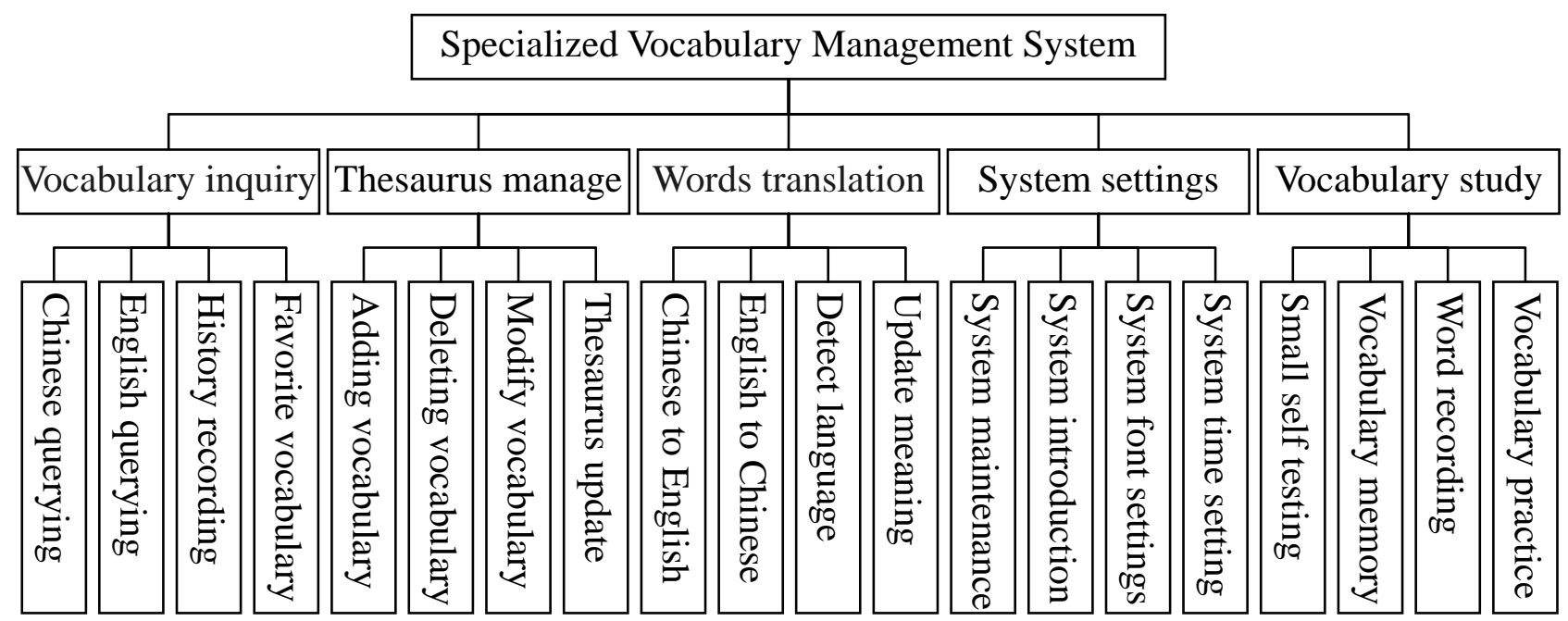

Fig. 2. System functional block diagram

\section{Detail Design}

Detail design is based on the outline design for the functionality of the system further deepening. The goal of detail design is twofold: the algorithm to implement the module function is logically correct and algorithm description should be concise and understandable. The detail design of system firstly needs to determine the design principles and methods, and the system is decomposed into several sub-systems, and then determines the objectives, functions and relationships contained in the various subsystems [8]. According to the characteristics of the software design and system requirements, design principles include the following aspects: (1) the principle of rationality, all designed in accordance with the requirements of the system as a principle, only to meet customer requirements is value, constantly try to figure out the system usability, which is a basic overview of reasonableness; (2) the principle of unity, if the design cannot be reached unity conditions, the architecture will constantly change and the iteration and replacement of versions will be confuse. (3) high-efficiency and high-performance principle, the so-called "high efficiency" refers to: the design work of systems should take into account the efficiency in the phase of system development and implementation, cannot occur repeated development or rework; and "high performance" refers to: system design should take into account the future performance of the system running whether meets the user's requirements; (4) safe and stable operation principle, any system software, ultimate requirement of the user is its ability to secure and stable operation, the system cannot respond and data losing or other issues frequently collapse should not occur, in addition, the user data privacy must be considered into safety category [9].

The overall design describes that the accounting English vocabulary management system contains vocabulary queries, thesaurus management, vocabulary translation, system settings and vocabulary study five functional modules. Vocabulary query module is mainly used for specialized 
accountancy English vocabulary query function, it can query Chinese specialized vocabulary and can also query English specialized vocabulary, and also has a historical memory function and collection function interested for vocabulary; thesaurus management module is the management for vocabulary databases, including vocabulary updating, adding, modifying, deleting and other management functions in the thesaurus; vocabulary translation module which functions like a small electronic dictionary, main function is translation for professional vocabulary, including Chinese to English and English to Chinese, and in this module it also includes detection function that automatic detection of inputting words, namely automatically detects the input vocabulary is English or Chinese, but also can improve the translation content to make meaning more accurate and perfect; system settings module is mainly to manage their own systems, including system maintenance, system functions introduction, vocabulary font settings and other functions; vocabulary learning module is mainly learning vocabulary, including to memory specialized vocabulary, self-tests on vocabulary, translation of some words and so on.

\section{Conclusion}

The development of technology and global integration make specialized English increasing people's attention. Combination of accounting and English as a class in the specialized English is a trend. The importance of accounting in today's society has become increasingly prominent and plays an irreplaceable role in the socio-economic sphere. With the accelerated process of economic globalization, accounting by the way of English extensively spreads and exchanges all around the globe. In the composition of foreign trade personnel, the accounting officer is clearly indispensable and plays increasingly important roles, which undoubtedly raise higher and higher requirements for the international business common language (English) capabilities of accounting staff. the accounting English vocabulary management system designed in the paper uses J2EE framework which has many advantages in technology, and the system has the function of almost meeting people's requirements for system, as well as operation and safety have good protection, for accounting English vocabulary learning provides a good platform and for learning accounting English has important significance.

\section{References}

[1] C. X. Cheng, "Features and Translation of Accounting English," Exam Magazine, vol. 3, no. 41, pp. 128-129, 2009.

[2] R. Yang, M. F. Lu, "Characteristic Analysis of English Vocabulary in Accounting Profession," Education Teaching Forum, vol. 6, no. 21, pp. 125-126, 2014.

[3] P. M. Cao, W. Zhao, Y. L. Xu, "J2EE Technology and Its Implementation," Computer Application, vol. 21, no. 10, pp. 21-23, 2012.

[4] L. L, "Research and Application of J2EE Frameworks and Design Patterns," Master's Degree of University of Electronic Science and Technology, 2007.

[5] Y. Y. Teng, "R\& D Three-Layers System Structure Based on J2EE," Liaoning Taxation College Journal, vol. 14, no. 5, pp. 36-37, 2002.

[6] Baidu Baike, "namespace," http://baike.baidu.com/view/228947.htm?fr=aladdin, 2014-11-4.

[7] P. H. Liu, "Design and Implementation of Supermarket Merchandise Sales Management System," Master's Degree of University of Electronic Science and Technology, 2013.

[8] Baidu Baike, "namespace," http://baike.baidu.com/view/228950.htm?fr=aladdin, 2014-11-4.

[9] Z. Yang, "Design and Implementation of Book Information Management System," Master's Degree of ShandongUniversity, 2013. 